\title{
The Rat Nervous System
}

\author{
by George Paxinos
}

Hardbound, 210x180 mm, 1056 pages, ISBN : 9780123742452; Academic Press Elsevier, 2014

www.elsevier.com

Anatomy 2014;8:47, (C) 2014 TSACA

The brain is one of the most distinguishing features of the human species, and understanding it is one of the greatest challenges of twenty first century science. Mice and rats are strikingly similar to humans in their anatomy, physiology and genetics - over $95 \%$ of the mouse genome is similar to human genome. Rodents are the dominant species used in neuroscience research; the majority of articles published in neuroscience are on the rat and mouse. In the general neuroscience journal group, these two species account for $45 \%$ of the published pages. There are important advantages to working with rodents; rodents typically live less than 1 year, and are easy to manipulate due to their small size. Also, they serve as excellent models to some human diseases. At this age, when the tools now available for studying the rodent brain are improving at a remarkable pace, understanding the details of the rat brain has become very important, as there are a multitude of challenges for methods used in human brain research.

The Rat Nervous System 4th edition has recently been published (2015) by Academic Press Elsevier. Some authors are the same with the 3rd edition of 2004; some chapters have new authors. The first edition came out in 1985, and the second edition in 1994. The book is prepared as 31 chapters organized in 7 sections: development; peripheral nervous system and spinal cord; brainstem and cerebellum; diencephalon, basal ganglia, amygdala, and septum; cortex; and systems (central autonomic system; pain system; gustatory system; the olfactory system; the vestibular system; auditory system; visual system and cerebral vascular system).
Chapters from the 3rd edition have been com-

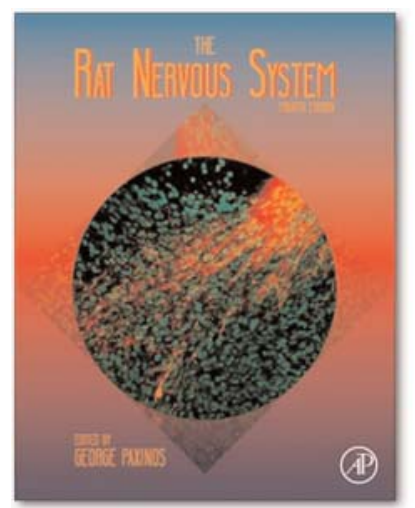
pletely revised and updated with findings of the last decade. The chapters provide a fundamental analysis of the anatomy of all areas of the central and peripheral nervous systems, with their structure, connections, and function explained in detail. They are well written, illustrated and referenced. Entirely new chapters have been added such as 'Neuromeric landmarks in the rat midbrain, diencephalon and hypothalamus, compared with acetylcholinesterase histochemistry', 'Tangential migration in the telencephalon' and "Organization of the basal forebrain cholinergic projection system".

The editor of the book, George Paxinos is from Neuroscience Research Australia and The University of New South Wales, Sydney, Australia. He is the author of almost 50 books on the structure of the brain of humans and experimental animals, including The Rat Brain in Stereotaxic Coordinates, now in its 7 th edition, the only neuroscience publication in the Thomson ISI 50 most cited items in the Web of Science.

This book will serve to researchers working not only with the rat as a model for brain anatomy, physiology, pharmacology, behavior, and genetics, but also to researchers working on other species, including humans, an indispensible guide definitely for all neuroscientists.

\footnotetext{
Review by Gülgün Şengül

Editor-in-Chief, "Anatomy”

Department of Anatomy, Faculty of Medicine,

Ege University, Bornova, 35100, Izmir, Turkey

e-mail: gulgun.sengul@gmail.com
} 